\title{
Modification Cross' Theorem on Triangle with Congruence
}

\author{
M. Rusdi Syawaludin*, Mashadi Mashadi, Sri Gemawati \\ Department of Mathematics, University of Riau, Pekanbaru, Indonesia \\ Email address: \\ rusdi.syawaludin@gmail.com (M. R. Syawaludin),mashadi.mat@gmail.com (M. Mashadi),gemawati.sri@gmail.com (S. Gemawati) \\ ${ }^{*}$ Corresponding author
}

\section{To cite this article:}

M. Rusdi Syawaludin, Mashadi Mashadi, Sri Gemawati. Modification Cross' Theorem on Triangle with Congruence. International Journal of Theoretical and Applied Mathematics. Vol. 4, No. 5, 2018, pp. 40-44. doi: 10.11648/j.ijtam.20180405.11

Received: December 29, 2018; Accepted: January15, 2019; Published: February 18, 2019

\begin{abstract}
Cross' theorem states if any triangle $A B C$, on each side constructed a square and vertices of the square are connected, it will form another triangle which has an equal area to triangle $A B C$. In this paper we will discuss the modification Cross' theorem on triangle, which is to construct a square outcase direction on each side triangle produced by Cross' theorem. The purpose of this paper is to modify Cross' theorem and give simple proof even junior or senior high school can answer. The process of proof is done in simple way, that is using a congruence approach. The result obtained are square vertices that are connected is form a trapezoid and have area 5 times the area of triangle $A B C$.
\end{abstract}

Keywords: Triangle, Square, Congruence, Cross' Theorem

\section{Introduction}

Geometry has been studied starting from elementary to college, one of the material is about plane. On plane we will know such as triangles, rectangles, squares or trapeziums. At the elementary school level students are just beginning to recognize the nature, elements and determine area, while at the middle and high school levels students have begun to understand concepts and theorems in mathematics.

One of the many theorems in geometry that discusses about triangles is the Cross' theorem. Cross' theorem states if it is any triangle $\mathrm{ABC}$ a square constructed on each side of triangle and a squares vertices are connected so it will form another triangle which has an area equal to the triangle $A B C$.

Cross' theorem discovered and named by 14 years old schoolboy David Cross, which was posed by Faux (2004). Cross' theorem can be proved by using congruence [7]. Congruence essentially means that two figures or objects are of the same shape and size which is has been studied at junior high school.

In general, this Cross' theorem only applies to triangles, but several authors have developed this Cross' theorem like Cross' theorem on the triangle using a rectangle and the Cross' Theorem on quadrilateral $[4,11]$. The results reveal that there are the relationship between the area area formed from the new triangles constructed and the initial triangle.
Various proofs Cross' theorem has also been described [2, 3]. Seeing the relationship of developed Cross' theorem, the authors are interested in modifying the cross theorem on a triangle, which is squares outcase direction constructed on each side triangle Cross' theorem, and a squares vertices connected it will form a trapezoid and has an area 5 times the area triangle $\mathrm{ABC}$, then if each side of trapezoid is extended until intersect with each other it will form another triangle and that triangle is congruent with triangle $\mathrm{ABC}$, shown in Figure 1.

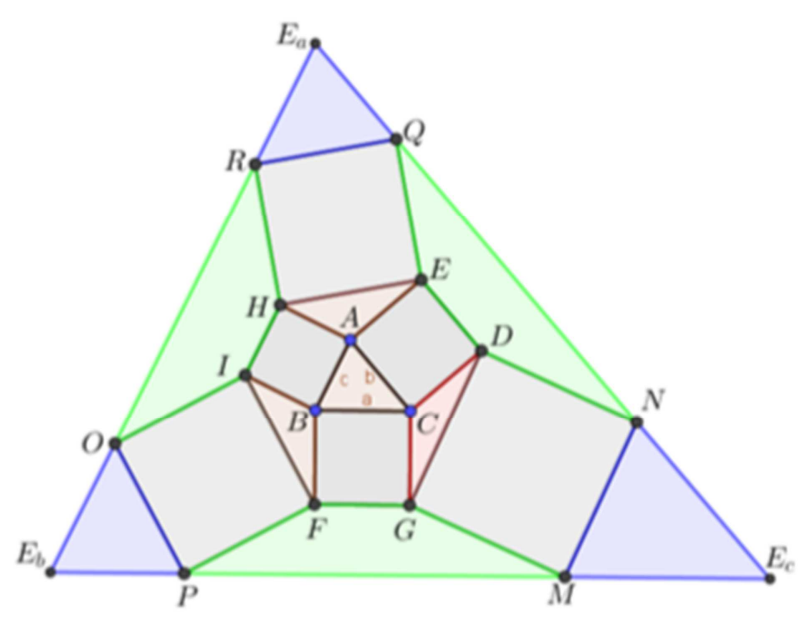

Figure 1. Illustration modification Cross' theorem. 
Lots of software that can be used in learning mathematics especially in geometry, One software which can be used is Geogebra. Geogebra application is dynamic mathematical software that can be used as a tool in mathematics learning. In this paper using software Geogebra which is very helpful in constructing points and lines. Geogebra is a versatile software for learning mathematics in schools and colleges, Geogebra is used as a media for demonstration and visualization, tools for finding mathematical concepts and preparing materials for teaching.

Based on the description above, the author discusses proof of modification Cross' theorem using easy material and can be discusses by middle or high school students.

\section{Cross' Theorem}

Cross' theorem was first put forward by Faux (2004) for reader of the Mathematics Theaching Journal. Faux states that a triangle $A B C$ on each sides triangle a squares are constructed, then a near square vertices connected it will form a new triangles and has an equal area to triangle $A B C$ [2].

This Cross' theorem was discovered by David Cross. In general Cross' theorem applies to triangles, but some authors have developed Cross' theorem on triangles using rectangles and Cross' theorem on quadrilateral $[4,11]$. Cross' theorem and some of the proofs has been discussed that triangles are formed has the same area from initial triangle [1, 3], as explained in Theorem 2.1.

Theorem 2.1. Let $A B C$ denote any triangle, and construct squares on each side of the triangle outward which is $A B I H$, $B C G F$ and $A C D E$. If line $E H, I F$ and $D G$ constructed, then will form $\triangle A E H, \triangle B I F$ and $\triangle C D G$ which has area same to $\triangle A B C$, shown in Figure 2.

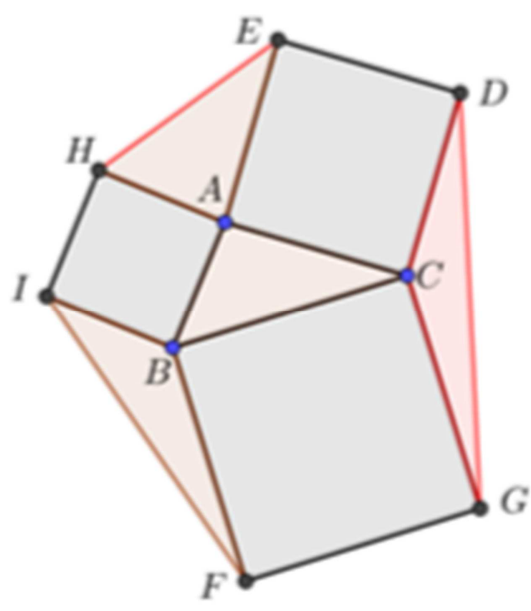

Figure 2. Illustration Cross' theorem

Proof. Suppose that $A B=c, B C=a$ and $A C=b$, using trigonometric formula to prove area triangle $A B C$, we have $L \triangle A B C=1 / 2 a c \sin \angle A B C=1 / 2 a b \sin \angle A C B=1 / 2 b c \sin$ $\angle B A C$. Consider $\triangle C D G$, since $\angle D C G=180-\angle A C B, C D=$ $b, C G=a$, hence area $\triangle C D G$

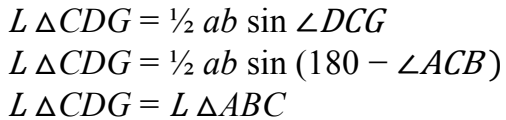

Similar way for $\triangle A E H$ and $\triangle B I F$, then $L \triangle C D G=L \triangle A E H=L \triangle B I F=L \triangle A B C$

This completes the proof.

Furthermore Cross' theorem using rectangles and not square on each side of the triangle it has been discussed in reference [4]. Because of many of rectangles that can be constructed, so conclude the side belongs to the rectangle must have the same proportion [4]. The result obtain is only new triangle has the same area, shown in Figure 3 and explained in Theorem 2.2.

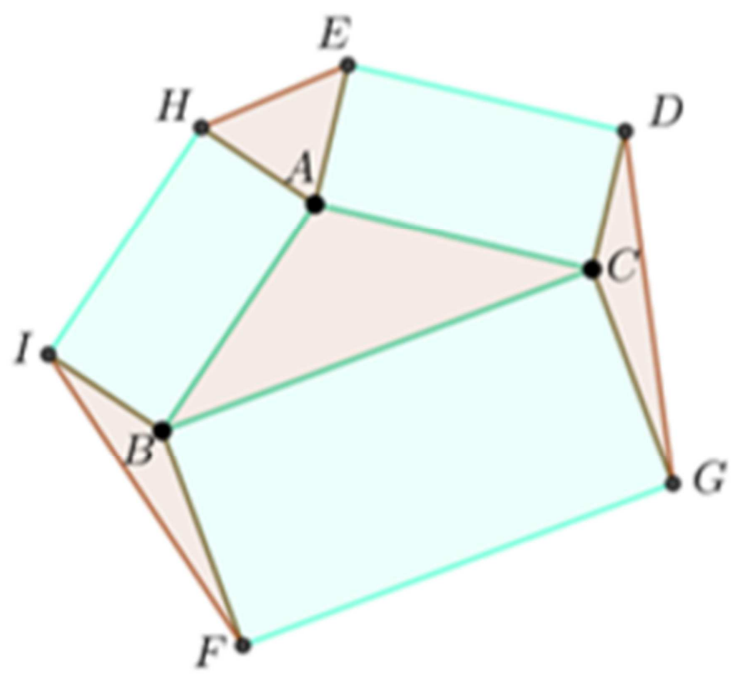

Figure 3. Illustration Cross' theorem using rectangles.

Theorem 2.2. Let $A B C$ denote any triangle, and consturct rectangles on each side of the triangle outward $A B I H, B C G F$ and $A C D E$ and has a width $1 / 2$ sides triangle. If line $E H, I F$ and $D G$ constructed, then will form $\triangle A E H, \triangle B I F$ and $\triangle C D G$ which has area same each other.

Proof. Let $A B=c, B C=a$ and $A C=b$, suppose each rectangle have same $r$ proportion, from Theorem 2.1 its easy to show

$L \triangle C D G=L \triangle A E H=L \triangle B I F=L \triangle A B C \times r^{2}$

This completes the proof.

Furthermore, Cross' theorem on a quadrilateral has been discussed in reeference [11], the theorem on a quadrilateral shows that sum of two pairs triangle at the opposite angle will be the same as the initial quadrilateral area, as explained in Theorem 3.3.

Theorem 3.3 Let $A B C D$ denote any quadrilateral, square construction on each side of the quadrilateral outward $A D G H, A B F E, B C L K$ and $C D J I$. If line $E H, F K, L I$ and $J G$ constructed, then will form $\triangle A E H, \triangle B F K, \triangle C L I$ and $\triangle J D G$ which has area $L \triangle A E H+L \triangle C I L=L A B C D$ and $L \triangle B F K+$ $L \triangle D G J=L A B C D$, shown in Figure 4. 


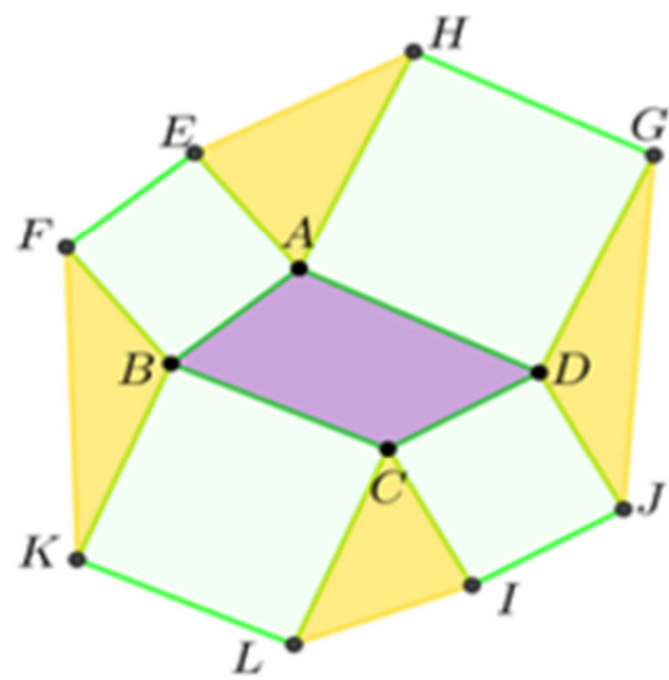

Figure 4. Illustration Cross' theorem on quadlilateral.

Many ideas of congruence concepts are discussed [5-7]. Then in this paper prove the modification Cross' theorem with concepts understood by junior and senior high school students the concept of congruence. The proof pattern in this paper is widely used [8-10]. Based on the Cross' theorem is constructing a square on the sides of a triangle so author is interested in doing modification by constructing a square on a triangle formed from the Cross' theorem.

\section{Modification Cross' Theorem}

Modification Cross' theorem is based on triangles formed from the Cross' theorem, then construction a square outward direction on each sides of the triangle, if the square vertex is connected it will form new shape, explained in Theorem 3.1.

Theorem 3.1 Let $A E H, B I F$ and $C D G$ are triangles formed from Cross' theorem triangle $A B C$. Furthermore, $E H R Q$, $I F P O$ and $D G M N$ is constructed on each side triangle Cross' theorem, if vertices a squares are connected then it will form a trapezoid and have area $5 \mathrm{~L} \triangle A B C$, shown in Figure 5.

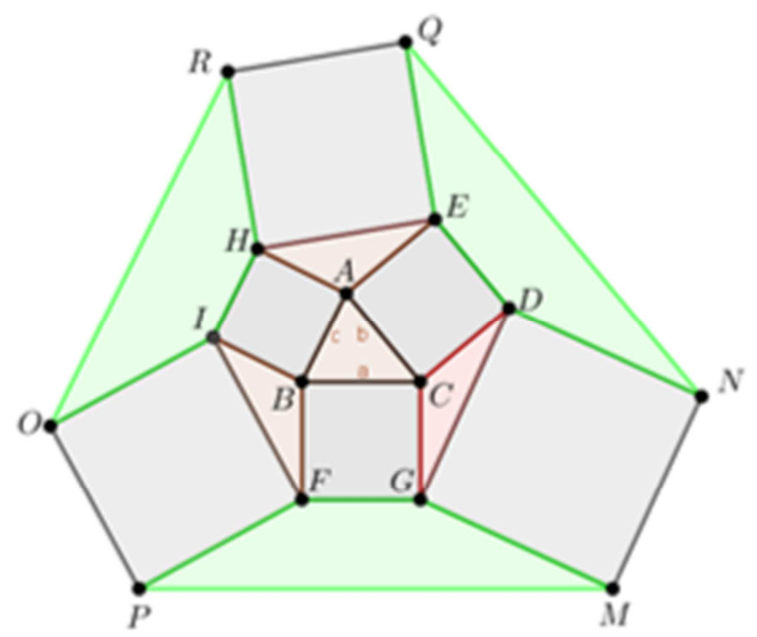

Figure 5. Illustration modification Cross'theorem.
Proof. Will be shown that FGMP, HIOR and DEQN are trapezoid and has an area $5 L \triangle A B C$. To show FGMP trapezoid it will proved $F G / / P M$. Suppose $A^{\prime}, D^{\prime}, I^{\prime}, P^{\prime}$ and $M^{\prime}$ respectively is projection of point $A$ to $B C, D$ to $G C, I$ to $F G$ and $M$ to $F G$ so that several triangle are formed its $\triangle A B A^{\prime}, \triangle C D D^{\prime}, \triangle B I I^{\prime}, \triangle F P P^{\prime}$ and $\triangle G M M^{\prime}$, as in Figure 6.

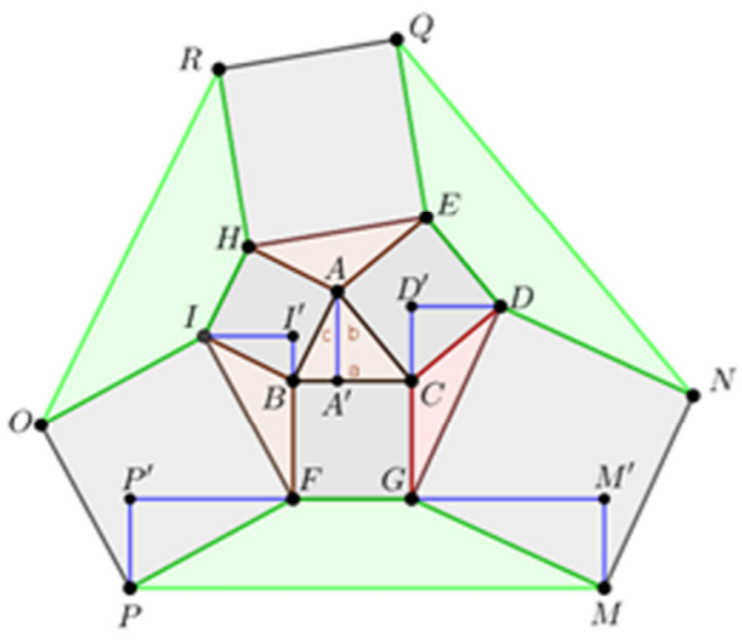

Figure 6. Illustration projection point in modification Cross' theorem.

Consider $\triangle I B F$ on Figure 6 , we have

$$
\begin{aligned}
& \angle I B F+\angle A B I+\angle A B C+\angle C B F=360 \\
& \angle I B F+90+\angle A B C+90=360 \\
& \angle I B F+\angle A B C+180=360 \\
& \angle I B F=360-180-\angle A B C \\
& \angle I B F=180-\angle A B C
\end{aligned}
$$

Thus on $\triangle I B F$ we have

$\angle I B I^{\prime}+\angle I B F=180$

$\angle I B F+(180-\angle A B C)=180$

$\angle I B I^{\prime}=180-(180-\angle A B C)$

$\angle I B I^{\prime}=\angle A B C$

Hence, for $\triangle B A A^{\prime}$ and $\triangle B I I^{\prime}$, since $\angle I B I^{\prime}=\angle A B A^{\prime}, \angle B A^{\prime} A$ $=\angle B I I^{\prime}=90^{\circ}$ and $B A=B I$ then $\triangle B A A^{\prime} \cong \triangle B I I^{\prime}$, so that

$$
\mathrm{AA}^{\prime}=\mathrm{II}^{\prime}
$$

For $\triangle C D^{\prime} D$ and $\triangle C A A^{\prime}$, with similar way obtained $\angle D C G$ $=180-\angle A C A^{\prime}$ and $\angle D C D^{\prime}=\angle A C A^{\prime}$, since $\angle D C D^{\prime}=$ $\angle A C A^{\prime}, \angle C D^{\prime} D=\angle C A^{\prime} A=90^{\circ}$ and $C D=C A$ then $\triangle C D D^{\prime} \cong$ $\triangle C A A^{\prime}$, so that

$$
\mathrm{DD}^{\prime}=\mathrm{AA}^{\prime}
$$

Next, on $\triangle G M M$ ' and $\triangle G D D^{\prime}$, with similar way obtained $\angle F G M=180-\angle C D G$ and $\angle M G M^{\prime}=\angle C G D$, since $\angle M G M^{\prime}$ $=\angle C G D, \angle G M^{\prime} M=\angle G D^{\prime} D=90^{\circ}$ and $G M=G D$ then $\triangle G M M^{\prime} \cong \triangle G D D^{\prime}$, so that

$$
\mathrm{MM}^{\prime}=\mathrm{DD}^{\prime}
$$

And then for $\triangle F P P^{\prime}$ and $\triangle F I I^{\prime}$, with similar way obtained $\angle G F P=180-\angle I F I^{\prime}$ and $\angle P F P^{\prime}=\angle I F I^{\prime}$, since $\angle P F P^{\prime}=$ $\angle I F I^{\prime}, \angle F P^{\prime} P=\angle F I^{\prime} I=90^{\circ}$ and $F P=F I$ then $\triangle F P P^{\prime} \cong$ $\triangle F I I^{\prime}$, so that 


$$
\mathrm{PP}^{\prime}=\mathrm{II}^{\prime}
$$

Based on (1), (2), (3) and (4) obtained

$$
\mathrm{MM}^{\prime}=\mathrm{DD}^{\prime}=\mathrm{AA}^{\prime}=\mathrm{II}^{\prime}=\mathrm{PP}^{\prime}
$$

Because $M M^{\prime}=P P^{\prime}$ then $P M / / P^{\prime} M^{\prime}$, so that $F G / / P M$.

Moreover, to calculate area $F G M P$, consider trapezoid $P^{\prime} M^{\prime} M P$ on Figure 6 , we have equation obtained

$$
\mathrm{PM}=\mathrm{P}^{\prime} \mathrm{M}^{\prime}=\mathrm{FG}+\mathrm{FP}^{\prime}+\mathrm{GM}^{\prime}
$$

Consider line $F P^{\prime}$, since $\triangle F P P^{\prime} \cong \triangle F I I^{\prime}$ and $\triangle B A A^{\prime} \cong$ $\triangle B I I^{\prime}$ then

$$
F P^{\prime}=F I^{\prime}=F B+B I^{\prime}=F B+B A^{\prime}
$$

Same for line $G M^{\prime}$, since $\triangle G M M^{\prime} \cong \triangle G D D^{\prime}$ and $\triangle C D D^{\prime}$ $\cong \triangle C A A^{\prime}$ then

$$
\mathrm{GM}^{\prime}=\mathrm{GD}^{\prime}=\mathrm{GC}+\mathrm{CD}=\mathrm{GC}+\mathrm{CA}^{\prime}
$$

Substitution (7) and (8) to (6), hence $P M=4 a$.

Next, suppose $L, K$ and $S$ each point that divides line $P M$ to be 4 parts and each one have distance $a$, shown in Figure 7.

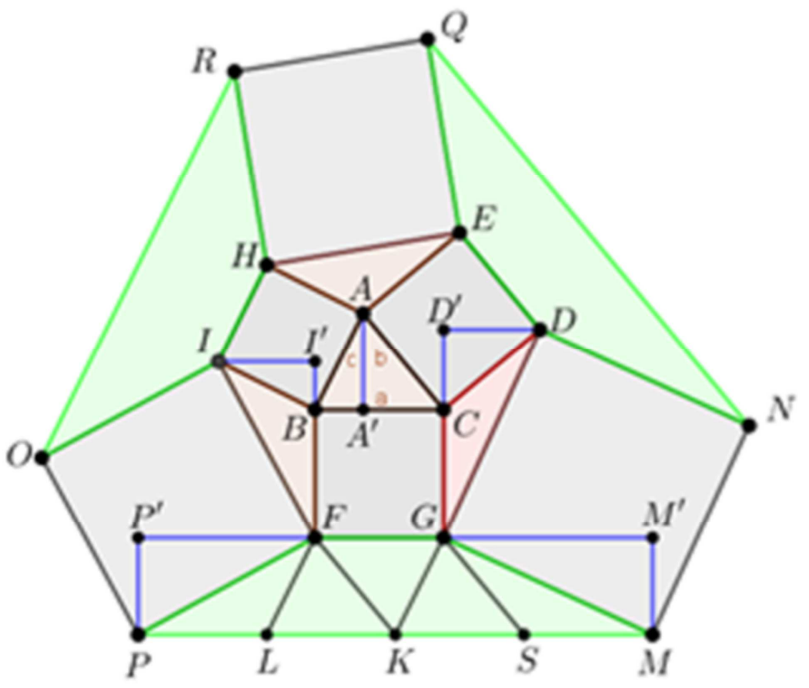

Figure 7. Illustration triangles on trapezoid FGMP.

Therefore, $P L=L K=K S=S M=a, P^{\prime} M^{\prime} / / P M$ and $P P^{\prime}=$ $A A^{\prime}=M M^{\prime}$ then obviously

$L \Delta P L F=L \Delta L K F=L \Delta F K G=L \Delta K S G=L \Delta S M G=$ $L \triangle A B C$,

Hence,

$L F G M P=5 L \triangle A B C$.

In similar way for $H I O R$, that is $H I / / R O$ and make that $H I O R$ trapezoid, then $R O=4 c$, hence 5 triangles will form in trapezoid $H I O R$ and each triangle have same area with triangle $A B C$, shown in Figure 8.

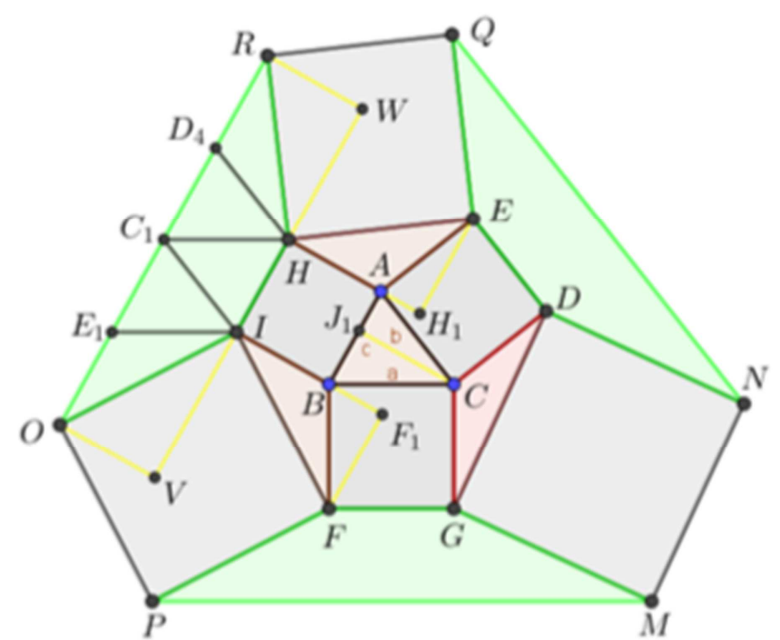

Figure 8. Illustration proof for HIOR.

Moreover, similar way apply for $D E Q N$ which is get $D E Q N$ is trapezoid and have area $5 L \triangle A B C$, shown in Figure 9.

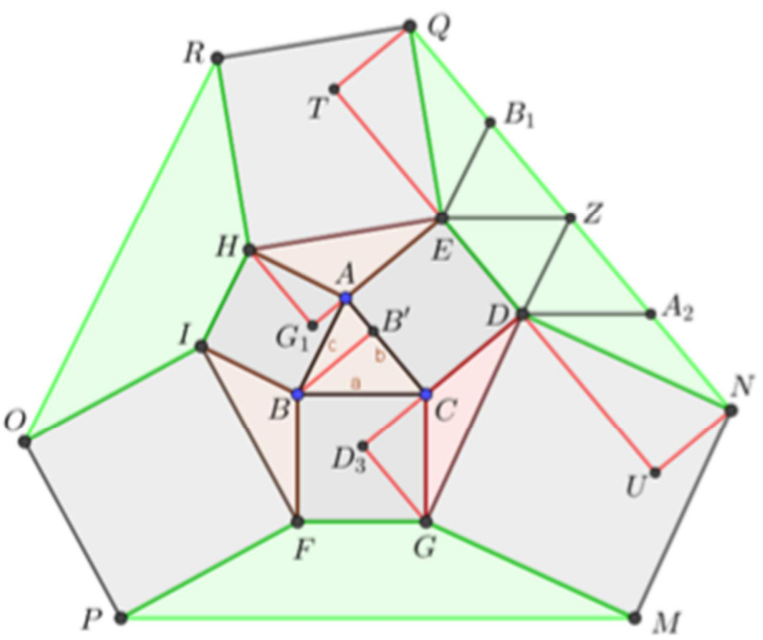

Figure 9. Illustration proof for $D E Q N$.

This completes the proof.

Next, will discuss about the extension of line on the sides of expansion trapezoid. In general, if the sides of the triangle produced by the Cross' theorem is extended so that it intersects, there is no relation between the new triangle formed with the initial triangle. Unique to this modification Cross' theorem, if the outer edges of the trapezoid is extended will form a new triangle that is uniform with the initial triangle, as explained in Theorem 3.2.

Theorem 3.2 Let FGMP, HIOR and DEQN is a trapezoid from modification Cross' theorem on triangle $A B C$, then suppose $E_{a}, E_{b}$ and $E_{c}$ are intersects each line $R O$ to $N Q, R O$ to $P M$ and $N Q$ to $P M$, hence will form $\triangle E_{a} E_{b} E_{c}$ which is uniform to $\triangle A B C$, shown in Figure 10. 


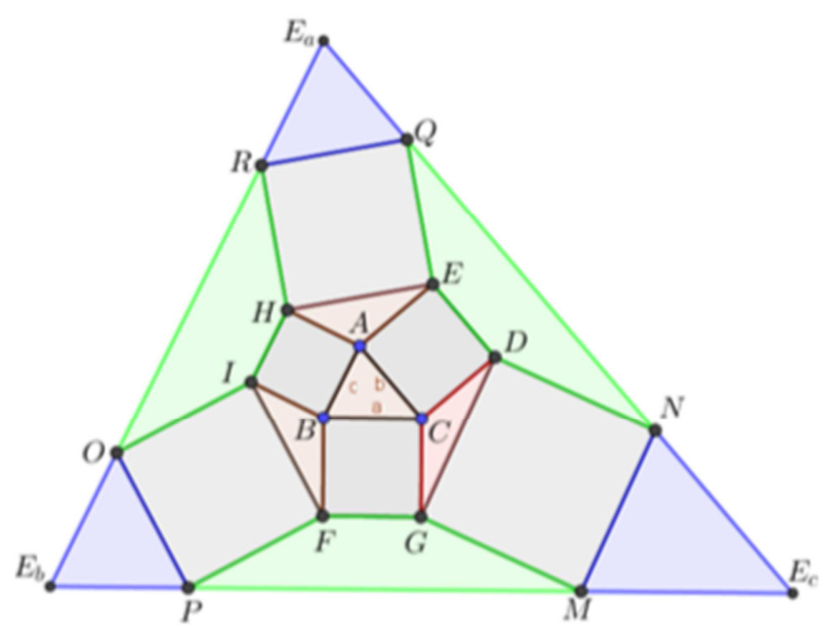

Figure 10. Illustration extended side of trapezoids.

Proof. To proof $\triangle E_{a} E_{b} E_{c} \approx \triangle A B C$ will show that each angle on triangle have same size. Therefore from proof Theorem 3.1 we have $F G / / P M$ so that $B C / / P M$ then on other side $A B / / R O$ and $A C / / N Q$. Because each line are parallel to each side then obviously the extension will also form a same angle which is $\angle E_{a} E_{b} E_{c}=\angle A B C, \angle E_{b} E_{c} E_{a}=\angle B C A$ and $\angle$ $E_{c} E_{a} E_{b}=\angle C A B$. Thus $\triangle E_{a} E_{b} E_{c} \approx \triangle A B C$.

\section{Conclusion}

In this paper the authors only discuss the shape formed in modification Cross' theorem on triangle and his area. In addition there is relationship betwen trapezoid and initial triangle at modification Cross' theorem on triangle. Therefore, further discussing could be focused on sum of area modification Cross' theorem on triangle include the square, and more expansion on the side of trapezoid.

\section{Acknowledgements}

I would like to express my gratitude to my advisor, Prof. Mashadi, Dr. M. D. H. Gamal and my mentors for their encouragement, constant support, helpful remarks and insightful comments on the paper.

\section{References}

[1] L. Baker and I. Harris, a Day to Remember Kath Cross. Mathematics Teaching, (2004), 189, 20-22.

[2] G. Faux, Happy 21st Birthday Cockcroft 243 and All The Other Threes, Mathematics Teaching, (2004), 189, 10-12.

[3] J. Gilbey, Responding to Geoff Fauxs Challenge, Mathematics Teaching, (2005), 190, 16.

[4] Manuel and Luis, Students' Development of Mathematical Practices Based on The Use of Computational Technologies, Center for Research and Advanced Studies, (2006).

[5] Mashadi, Geometri Lanjut, UR Press, Pekanbaru, (2015).

[6] Mashadi, Geometri: Edisi Kedua, UR Press, Pekanbaru, (2015).

[7] Mashadi, Pengajaran Matematika, UR Press, Pekanbaru, (2015).

[8] Mashadi, C. Valentika and S. Gemawati, Developtment of Napoleon on the Rectangles in Case of Inside Direction", International Journal of Theoretical and Applied Mathematics, (2017), 3 (2), 54-57.

[9] C. Valentika, Mashadi and S. Gemawati, The Development of Napoleons Theorem on The Quadrilateral in Case of Outside Direction, Pure and Applied Mathematics Journal, (2017), 108-113.

[10] C. Valentika, Mashadi and S. Gemawati, the Development of Napoleons Theorem on Quadrilateral with Congruence and Trigonometry, Bulletin of Mathematics, (2017), 8 (01), 97108 .

[11] Viliers, An Example of Discovery Function of Proof, Mathematics in School, (2017), 36 (4), 9-11. 